\title{
Effect of Implementing Nursing Care Guidelines on the Occurrence of Deep Vein Thrombosis among Orthopedic Patients
}

\author{
Awatef Abdel Hamid Mohamed ${ }^{1}$, Walaa Nasreldin Othman ${ }^{2}$, \\ Barakat Sayed El Alphy ${ }^{3}$ Amany Mohamed Sheble ${ }^{4}$ \\ ${ }^{1}$ Assistant Lecturer at Medical-Surgical Nursing, Faculty of Nursing, Mansoura University, Egypt \\ ${ }^{2}$ Lecturer of Medical surgical Nursing, Faculty of Nursing, Mansoura University, Egypt \\ ${ }^{3}$ professor of Orthopedic surgery, Faculty of Medicine, Mansoura University, Egypt \\ ${ }^{4}$ professor of Medical surgical Nursing, Faculty of Nursing, MansouraUniversity, Egypt
}

\begin{abstract}
:
Background: Pulmonary embolism is the main cause of mortalities in lower limb Deep Venous Thrombosis (DVT). It leads to yearly 50-200 thousand deaths in the United States (US). Hence, DVT is an important condition that needs to be considered for the safe care of hospitalized patients to avoid related morbidities and mortalities

Aim of the current study was to evaluate the effect of implementing nursing care guidelines on the occurrence of deep vein thrombosis among orthopedic patients

Method; causal comparative research design used in carrying out this study that was conducted at orthopedic departments of Mansoura Emergency Hospital. A convenience sample of participants was used over a period of 6 months who correspond to inclusion criteria. Tools used for data collection in this study consisted of three tools as the following; first tool; composed of three parts, socio-demographic characteristics, DVTrisk factors scale, and : Patient medical data. The second tool is concerned with assessment of nurses' performance regarding application of DVT preventive guidelines; and the third tool is Deep vein thrombosis evaluation sheet.

Results: The present study revealed that the intervention is beneficial in terms of patient outcomes, with significantly lower DVT risk grade and score compared with the control group patients and improving the nurses performance regarding the application of DVT preventive guidelines

Conclusion; implementing the designed nursing guidelines for DVT prevention decrease the incidence of DVT in the group followed by guidelines

Recommendation: continued nursing education about deep vein thrombosis prevention among orthopedic patients for nurses recommended to be organized regularly.
\end{abstract}

Keywords: Nursing care, DVT, Orthopedic patients

\section{Introduction}

The great concerns related assigned to the problem of DVT is mainly due to its outrageous consequences that may end with fatality. The main consequences are chronic lower limb problems caused by post-thrombotic syndrome (PTS), and pulmonary hypertension, which may become chronic. However, the most dread consequence of DVT remains the pulmonary embolism, which although less frequent, is life- threatening \{1\} Orthopedic surgery is an established risk factor for DVT. Major orthopedic surgery, such as THR, TKR, and hip fracture operations, is associated with a significant risk of postoperative VTE \{2\} The thromboplastin released from the soft tissue and bone during the process of dissection increases the risk for high rates of DVT. Added to this is the venous stasis during the operation, as well as the postoperative bed recumbence $\{\mathbf{3}\}$.

The annual incidence of DVT after major orthopedic surgery of the lower extremities, after age and gender adjustment was 70.67 per 100000 persons/years in Korea, with a trend of annual increase over the 5-year period studied. Moreover, the incidence rates of postoperative DVT for knee and hip replacement arthroplasty, and hip fracture operations were $0.22 \%, 0.15 \%$, and $0.16 \%$ respectively in the same study $\{4\}$. Additionally, Elective Total Knee Arthroplasty (TKA) was associated with an incidence rate of DVT of $1.3 \%$ in subjects who were admitted to intensive care units $\{\mathbf{5}\}$.

Therefore, the application of proper thrombo $า$-prophylaxis is crucial in the management of the patients who undergo major orthopedic operations. Nonetheless, no prophylaxis guidelines are yet established, and there is a orthopedic operations \{6\}Tool in order to recognize the patients at high risk of DVT is essential in order to be able to apply proper preventive measures early enough to mitigate the associated morbidities and mortalities 


\subsection{Aim;}

\section{Methodology}

To evaluate the effect of implementing nursing care guidelines on the occurrence of deep vein thrombosis

2.2 Research hypothesis;

The occurrence of deep venous thrombosis will be reduced in orthopedic patients who are followed by evidence based guidelines in comparison to orthopedic patients who are not followed by these guidelines

\subsection{Research design;}

A causal comparative design was used in this study.

\subsection{Setting;}

The orthopedic care unit of Mansoura emergency Hospital.

\subsection{Participants;}

The study subjects consisted of two groups, namely nurses and patients.60 patients divided randomly into two groups (30 patients in each group) and 60 nurses who provide direct nursing care for the patients

\subsection{Tools;}

Three tools were used for data collection in this study as the following;

2.6.1 Tool I: Patient's Assessment Data Sheet for DVT

This tool aims to assess the patients' risk factors for development of DVT and it consisted of three parts

Part I: Patient personal data: such as name, age, gender, educational level, marital. Status, diagnosis, family history, etc

PartII: Part II: DVT Risk Factors Scale: This part was used to identify patient's risk factors for developing DVT. It is composed of seven categories of risk factors

Part III Part III: Patient medical data: This was used to record information regarding the type of anesthesia administered, the patient hydration state, and the types of medications used with special emphasis on salicylates, anticoagulants, and thrombolytic agents.

\subsubsection{Tool II:}

This tool was used to assess this performance of the nurses concerning application of elastic stockings with and leg elevation and range of motion exercise. These two nursing procedures are considered effective in the prevention of DVT. It included two checklists, in addition to a section for the nurse's characteristics such as age, gender, nursing, qualification, experience years, and attendance of training courses. Nurse's characteristics such as age, gender, nursing, qualification, experience years, and attendance of training courses

- Application of elastic stockings: consisted of 16 steps, with four sub-steps. Each step was checked. As done or not done. These are scored one and zero respectively. The scores of all the steps are summed-up and converted into a percent score. The nurse achieving $80 \%$ or more is considered as having adequate performance while a total score less than $80 \%$ is considered inadequate performance.

- Range of motion exercises'. This consisted of five types of exercises each with a variable number of steps. Each step was checked as done or not done. These are scored one and zero respectively. The scores of all the steps are summed-up and converted into a percent score. A total score of $80 \%$ or more is considered as adequate performance while a total score less than $80 \%$ is considered inadequate performance.

A total score of nurse's performance was calculated by adding the sum scores of both elastic stockings and range, of motion. The steps are summed-up and converted into a percent score. A total score of $80 \%$ or more is considered as adequate performance.

\subsubsection{Tool III: Tool III: Deep vein thrombosis evaluation sheet}

This tool was modified by the researcher to assess the development of DVT in order to evaluate the effectiveness of the application of the nursing guidelines. It included three parts:

-Part I: Deep Vein Thrombosis Manifestations: This part was used to assess DVT manifestations. It assessed the patient for the presence of any of the five items: calf pain, calf tenderness, calf circumstance, skin color, and distension and warmth of the calf

-Part II: Laboratory Tests: This part was for recording patient's results of the laboratory tests to determine the coagulation status, whether in hyper-coagulation or not, as partial thrombin time (PTT), prothrombin time (PT), hemoglobin $(\mathrm{Hb})$, platelets and fibrinogen level

-Part 3: Doppler Ultrasound: This test is done to assess the blood supply in the veins of the lower limbs. The Doppler findings indicate the occurrence of DVT.

\subsection{Validity and reliability of the instruments}

The developed tool was tested for content- related validity by 10 experts, from the faculty of nursing and from the faculty of medicine, Mansoura University, who reviewed the tool for clarity, relevance, understanding, and applicability for implementation. According to their critiques, minor modifications were done. 
3.8 Pilot study

A pilot study was carried out on 5 patients and 5 from the orthopedic care unit of Mansoura Emergency hospital to assess the clarity and the applicability of the tool, and the necessary modification was done prior to data collection. Those patients were excluded in the main study.

\subsection{Ethical considerations:}

The researchers obtained the required permissions from the Research Ethics Committee of Nursing faculty, Mansoura University. Oral approval for the study was attended from each participant after verbal explanation of the study nature and objective were reported to all research participants. All patients were informed about their rights to agree or disagree to participate in the study and they were permitted to leave from the research at any point of time and this will not affect their care.

\subsection{Operational Design \\ Preparatory phase}

During this phase, the researcher reviewed pertinent literature to help in the development of the data collection tools as well as the intervention guidelines. Once developed, the tools were rigorously revised by ten experts, both from related nursing and medical disciplines, to ensure their face and content validity. All necessary modifications were done accordingly.

\section{Field of work}

Once the oral approval for the study was obtained, the researcher visited the study settings and met with the nurses and patients and explained the purpose of the study to them. Those who gave their consent and met the eligibility, criteria were incorporated in the study sample. The field of work was executed through assessment, planning, intervention, and evaluation phases.

Assessment phase: This phase included 34 nurses who served to determine the educational needs upon- which the training guidelines will be based. The data collected in this phase were analyzed, and the results demonstrated complete deficiency of performance of application of elastic stockings and of the range of motion exercises among the nurses. These findings were the baseline for construction of the study intervention.

Preparation phase: During this phase, the researcher developed the DVT nursing guidelines based on the assessment data and in the light of related literature. The preparation of the guidelines involved the following steps:

Determination the need and scope of the guidelines: The need was determined by the researcher from the current nursing practices obtained in the assessment phase, which identified the nurses' needs training as well as the available resources to implement the guidelines. Once the scope was determined, the researcher formulated the structured clinical questions that help to identify the evidence needed from the relevant systematic reviews. The formulated question was: "will the implementation of the nursing guidelines for prevention of DVT improve the clinical outcomes among orthopedic patients? Then, the researcher accurately defined:

The target population: all adult patients with lower limb orthopedic surgeries, who thus may have a high risk for developing DVT.

The health care setting: El-Mansoura Emergency Hospital.

The area: orthopedic care units.

Establishment of a multidisciplinary group for guidelines development: In addition to the researcher, three academic experts in medical-surgical nursing and vascular surgery convened to review the process of guidelines development and modification.

Identification of guidelines purpose and target. audience: As reflection of the multidisciplinary nature of the DVT preventive measures, the guidelines were aimed at medical and nursing staff. The purpose was to standardize a team approach and keep the patients at high risk for DVT in the center of care. This concept was considered in the development and modification of the preventive measures guidelines. The guidelines development group decided to formulate a DVT preventive measures team to be responsible for guidelines implementation.

Identification of the health outcomes: Specific health outcomes were selected. They included the absence of DVT manifestations and patent blood supply in the lower limbs. Once developed, the guidelines were piloted on a 5 nurses and on 5 patients over a ten-day period to test them

\subsection{Statistical analysis;}

After collection of the data, it was analyzed using the statistical package of social science "SPSS" software. Descriptive statistics in the form of frequencies and percentages for qualitative data, and means and standard deviations and medians and inter quartile ranges for quantitative variables. Quantitative continuous data were compared using the non- parametric Mann-Whitney test. Qualitative categorical variables were compared using 
chi-square test. Whenever the expected values in one or more of the cells in a $2 \times 2$ tables was less than 5, Fisher exact test was used instead. In larger than $2 \times 2$ cross-tables, no test could be applied whenever the expected value in $10 \%$ or more of the cells was less than 5 . Statistical significance was considered at p-value $<0.05$.

\section{Results}

Table 1: frequency distribution of socio-demographic characteristics and health relevant data $(n=63)$

Table (1) illustrates that more than half of the participants (54\%) aged from 50 to 60 years with mean age 47.3. The highest proportions of study participants were female (68.3\%), non-educated (69.8\%), not working $(71.4 \%)$ and non-smoker $(88.9 \%)$. In relation to surgically treated joint, right hip $(36.5 \%)$ was the commonest. Finally, regarding the reason for surgical replacement, around two-third of patients $(63.5 \%)$ suffered from osteoarthritis. As regards the socio-demographic characteristics of the patients in the study and control groups in the intervention phase samples, Table 4 indicates no statistically significant differences. They had exactly the same median age of 39 years. The sexwas almost equal or equal in the two groups. As for their level of education, the majority were having basic or diploma degrees.

Table 1:Personal characteristics of patients in the study and control groups

\begin{tabular}{|c|c|c|c|c|c|c|}
\hline & \multicolumn{4}{|c|}{ Group } & \multirow{3}{*}{$\begin{array}{c}X^{2} \\
\text { Test }\end{array}$} & \multirow{3}{*}{$\begin{array}{c}\text { li } \\
\text { vable }\end{array}$} \\
\hline & \multicolumn{2}{|c|}{$\begin{array}{l}\text { Study } \\
(n=30)\end{array}$} & \multicolumn{2}{|c|}{$\begin{array}{l}\text { Control } \\
(n=30)\end{array}$} & & \\
\hline & No. & $\%$ & No. & $\%$ & & \\
\hline Age: & & & & & & \\
\hline$<35-$ & 4 & 13.3 & 4 & 13.3 & & \\
\hline $35+$ & 26 & 86.7 & 26 & 86.7 & Fisher & 1.00 \\
\hline Range & \multirow{3}{*}{\multicolumn{2}{|c|}{$\begin{array}{c}29.0-54.0 \\
41.4+6.8 \\
39.00\end{array}$}} & \multirow{3}{*}{\multicolumn{2}{|c|}{$\begin{array}{c}29.0-55.0 \\
41.8+7.4 \\
39.00 \\
\end{array}$}} & & \\
\hline Mean \pm SD & & & & & $\mathrm{t}=0.00$ & 0.97 \\
\hline Median & & & & & & \\
\hline \multicolumn{7}{|l|}{ Gender: } \\
\hline Male & 16 & 53.3 & 15 & 50.0 & & \\
\hline Female & 14 & 46.7 & 15 & 50.0 & 0.07 & 0.80 \\
\hline \multicolumn{7}{|l|}{ Education: } \\
\hline Illiterate & 8 & 26.7 & 8 & 26.7 & & \\
\hline \multirow{2}{*}{$\begin{array}{l}\text { Basic/diploma } \\
\text { University }\end{array}$} & 20 & 66.7 & 22 & 73.3 & - & - \\
\hline & 2 & 6.7 & 0 & 0.0 & & \\
\hline
\end{tabular}

Table (2) Comparing the Deep Venous Thrombosis (DVT) risk factors in the study and control, This table points to few statistically significant differences. Thus, the patients in the study group had significantly higher risks due to pelvic surgical operations $(\mathrm{p}=0.007)$ and anemia $(\mathrm{p}=0.001)$. Conversely, the patients in the control group had higher risk due to pregnancy/puerperium $(\mathrm{p}=0.04)$ and varicose veins $(\mathrm{p}<0.001)$. None of the other risk factors showed statistically significant differences between the two groups.

Table 2: Risk factors for DVT between patients in the study and control groups

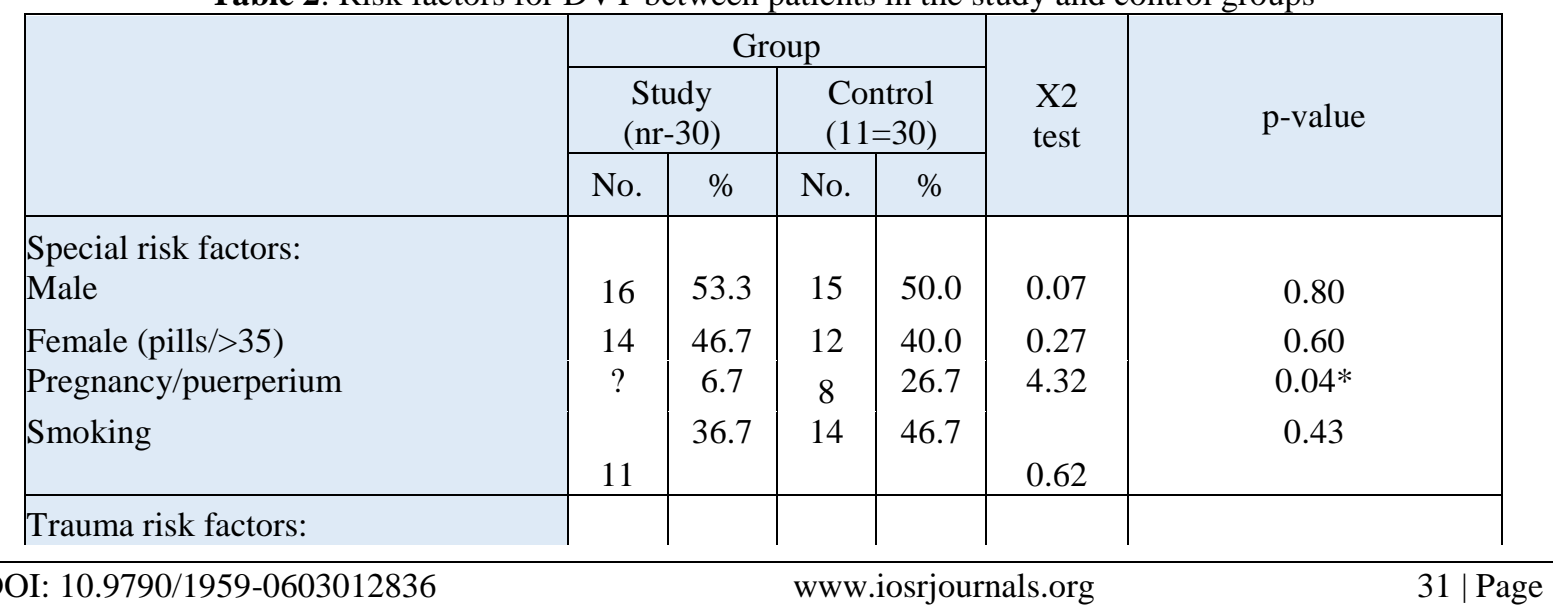




\begin{tabular}{|l|c|c|c|c|c|c|}
1 head/neck & 2 & 6.7 & 1 & 3.3 & Fisher & 1.00 \\
Chest & 1 & 3.3 & 0 & 0.0 & Fisher & 1.00 \\
Chest \& neck & 2 & 6.7 & 0 & 10.0 & Fisher & 1.00 \\
Spinal & 4 & 13.3 & 3 & 10.0 & Fisher & 1.00 \\
Pelvic & 16 & 53.3 & 17 & 56.7 & 0.07 & 0.80 \\
Lower limbs & 21 & 70.0 & 23 & 76.7 & 0.34 & 0.56 \\
\hline Surgical operation: & & & & & & \\
None & 8 & 26.7 & 1 & 3.3 & & \\
Pelvic & 8 & 26.7 & 4 & 13.3 & 9188 & $0.007^{*}$ \\
Orthopedic/Spinal & 14 & 46.7 & 25 & 83.3 & & \\
High risk diseases: & & & & & & \\
Nephritic syndrome & 1 & 3.3 & 0 & 0.0 & Fisher & 1.00 \\
Ulcerative colitis & 9 & 30.0 & 11 & 36.7 & 0.30 & 0.58 \\
Anemia & 11 & 36.7 & 1 & 3.3 & 10.42 & $0.001^{*}$ \\
Sickle cell & 5 & 16.7 & 9 & 30.0 & 1.49 & 0.22 \\
Polycythemia & 0 & 0.0 & 1 & 3.3 & Fisher & 1.00 \\
Hemolytic & 0 & 0.0 & 0 & 0.0 & 0.00 & 1.00 \\
Congestive heart failure & 0 & 0.0 & 0 & 0.0 & 0.00 & 1.00 \\
Myocardial infarction & 0 & 0.0 & 0 & 0.0 & 0.00 & 1.00 \\
Malignancy & 5 & 16.7 & 5 & 16,7 & 0.00 & 1.00 \\
Hyper-coagulation & 13 & 43.3 & 19 & 63.3 & 2.41 & \\
Varicose veins & & & & & & 0.12 \\
Stroke & 1 & 3.3 & 17 & 56.7 & 20.32 & $<0.001^{*}$ \\
& & & & & & \\
& 0 & 0.0 & 0 & 0.0 & 0.00 & 1.00 \\
\hline
\end{tabular}

High $(17+) \square$ Low $(<17)$
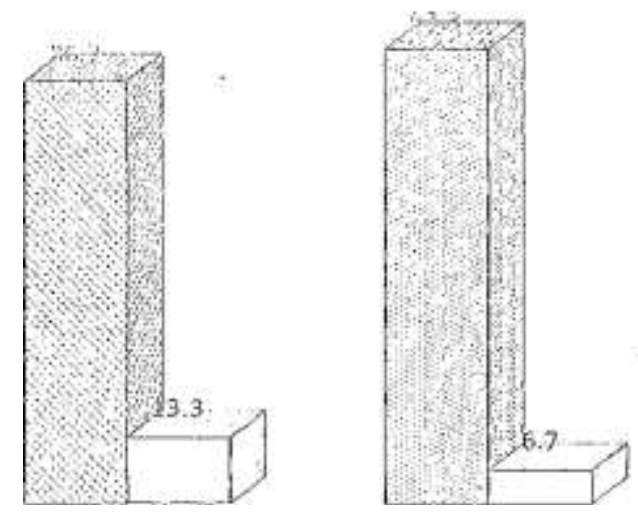

Figure 1: Scores of risk factors for DVT between patients in the study and control groups

Figure (1)determines that the majority of the patients in the study and control groups were at high risk of DVT, and no statistically significant difference between the two groups $(\mathrm{p}=0.67)$

Table (3) points to statistically significant differences between the nurses in the study and control groups in their total performance of application of elastic stockings $(\mathrm{p}<0.001)$, range of motion exercises $(\mathrm{p}<0.001)$, and total practice $(\mathrm{p}<0.001)$. It can be noticed that all $(100 \%)$ the nurses in the study group had adequate practice after the intervention compared to none $(0.0 \%)$ in the study group. 
Table 3: Total performance between nurses in the both groups

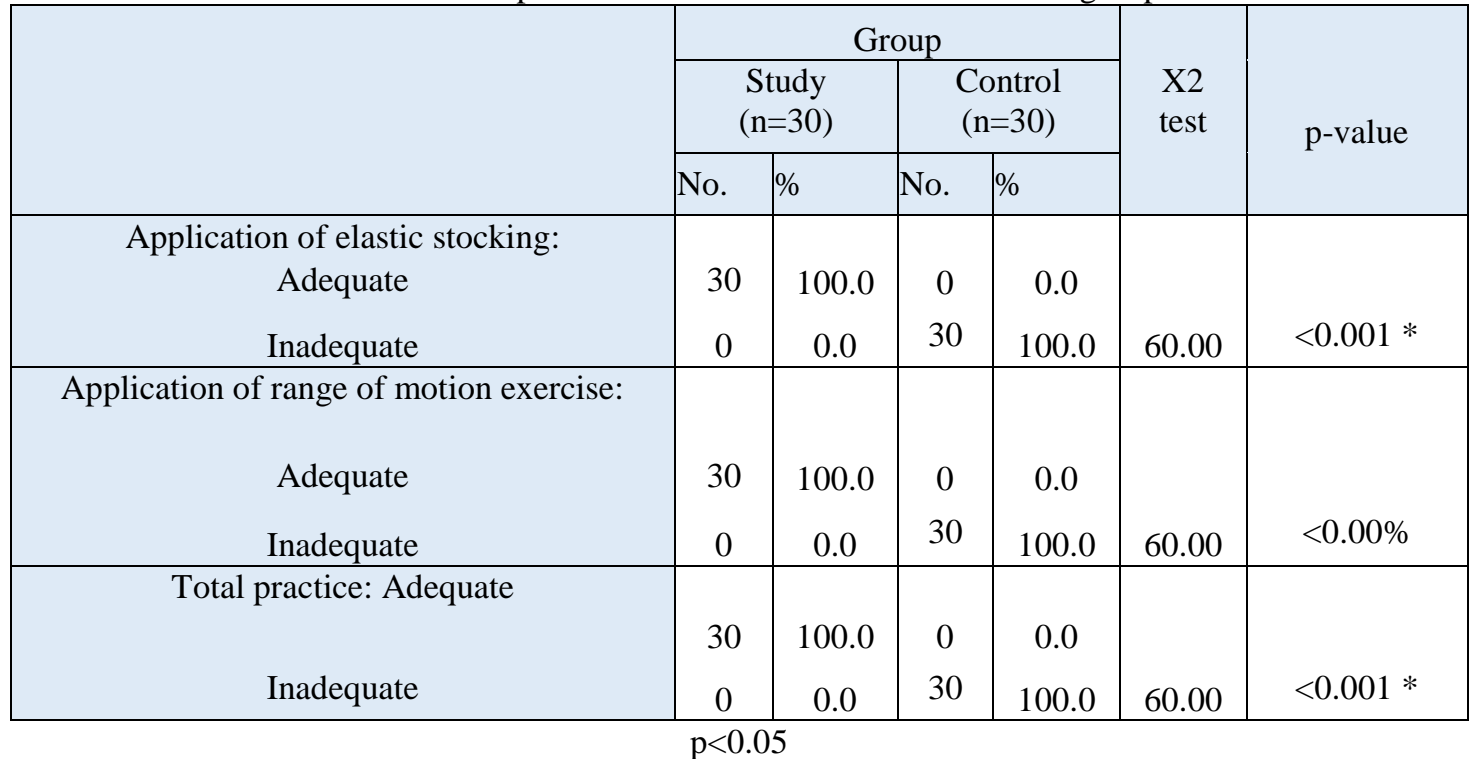

Table (4)Likewise, the comparison of the total DVI scores risk among patients in the both groups throughout the 14-day follow-up (Table 4) revealed statistically significant dissimilarities on the fifth $(\mathrm{p}=0.045)$ and sixth $(\mathrm{p}=0.02)$ days. It is noticed that the mean and median scores of the patients inthe control group were higher compared with those among the patients in the studygroup

Table 4: Comparison of DVT risk scores among patients in the study and control groups throughout the 14-day follow-up

\begin{tabular}{|c|c|c|c|c|c|c|}
\hline & \multicolumn{2}{|c|}{ Study $(n=30)$} & \multicolumn{2}{|c|}{ Control $(n=30)$} & \multirow{2}{*}{$\begin{array}{c}\text { Mann } \\
\text { Whitney } \\
\text { Test }\end{array}$} & \multirow[t]{2}{*}{ p-valuc } \\
\hline & Mean \pm SD & Median & Mean \pm SD & Median & & \\
\hline Day 1 & $20.5 * 2.9$ & 20.50 & $20.9 * 2.3$ & 22.00 & 0.79 & 0.37 \\
\hline Day 2 & $20.1 \pm 2.6$ & 20.00 & $20.9 * 2.3$ & 22.00 & 1.98 & 0.16 \\
\hline Day 3 & $20.1 \pm 2.6$ & 20.00 & $20.9 * 2.3$ & 22.00 & 1.98 & 0.16 \\
\hline Day 4 & $20.1 \pm 2.6$ & 20.00 & $20.9 * 2.3$ & 22.00 & 1.98 & 0.16 \\
\hline Day 5 & $20.1 * 2.6$ & 20.00 & $23.6 * 6.7$ & 22.00 & 4.02 & $0.045^{*}$ \\
\hline Day 6 & $20.3 \pm 3.1$ & 20.00 & $23.7 * 6.1$ & 22.00 & 5.61 & $0.02 *$ \\
\hline Day 7 & $20.3 * 3.1$ & 20.00 & $20.9 * 2.3$ & 22.00 & 1.52 & 0.22 \\
\hline Day 8 & $20.3 * 2.6$ & 20.50 & $21.4 * 4.0$ & 22.00 & 1.11 & 0.29 \\
\hline Day 9 & $20.1 * 2.6$ & 20.00 & $21.7 * 5.1$ & 22.00 & 2.30 & 0.13 \\
\hline Day 10 & $20.1 * 2.6$ & 20.00 & $20.9 * 2.3$ & 22.00 & 1.98 & 0.16 \\
\hline Day 11 & $20.1 * 2.6$ & 20.00 & $20.9 * 2.3$ & 22.00 & 1.98 & 0.16 \\
\hline Day 12 & $20.1 * 2.6$ & 20.00 & $20.9 * 2.3$ & 22.00 & 1.98 & 0.16 \\
\hline Day 13 & $20.1 * 2.6$ & 20.00 & $20.9 * 2.3$ & 22.00 & 1.98 & 0.16 \\
\hline Day 14 & $20.1 * 2.6$ & 20.00 & $22.4 * 6.4$ & 22.00 & 2.44 . & 0.12 \\
\hline
\end{tabular}

$\mathrm{p}<0.05$

As for the values of the laboratory tests, Table 5 demonstrates that on Day 1, the patients in the study group had significantly higher mean thromboplastin time $(\mathrm{p}=0.001)$, and lower means of clotting time $(\mathrm{p}<0.001)$, hemoglobin $(\mathrm{p}=0.03)$, and platelet count $(\mathrm{p}<0.001)$. On Day 14 , they continued to have significantly lower means of clotting time $(\mathrm{p}<0.001)$ and platelet count $(\mathrm{p}=0.002)$, in addition to lower hematocrit $(\mathrm{p}=0.008)$. 
Table 5: Comparison of laboratory tests among patients in the study and control groups in the first and $14^{\text {th }}$ clays

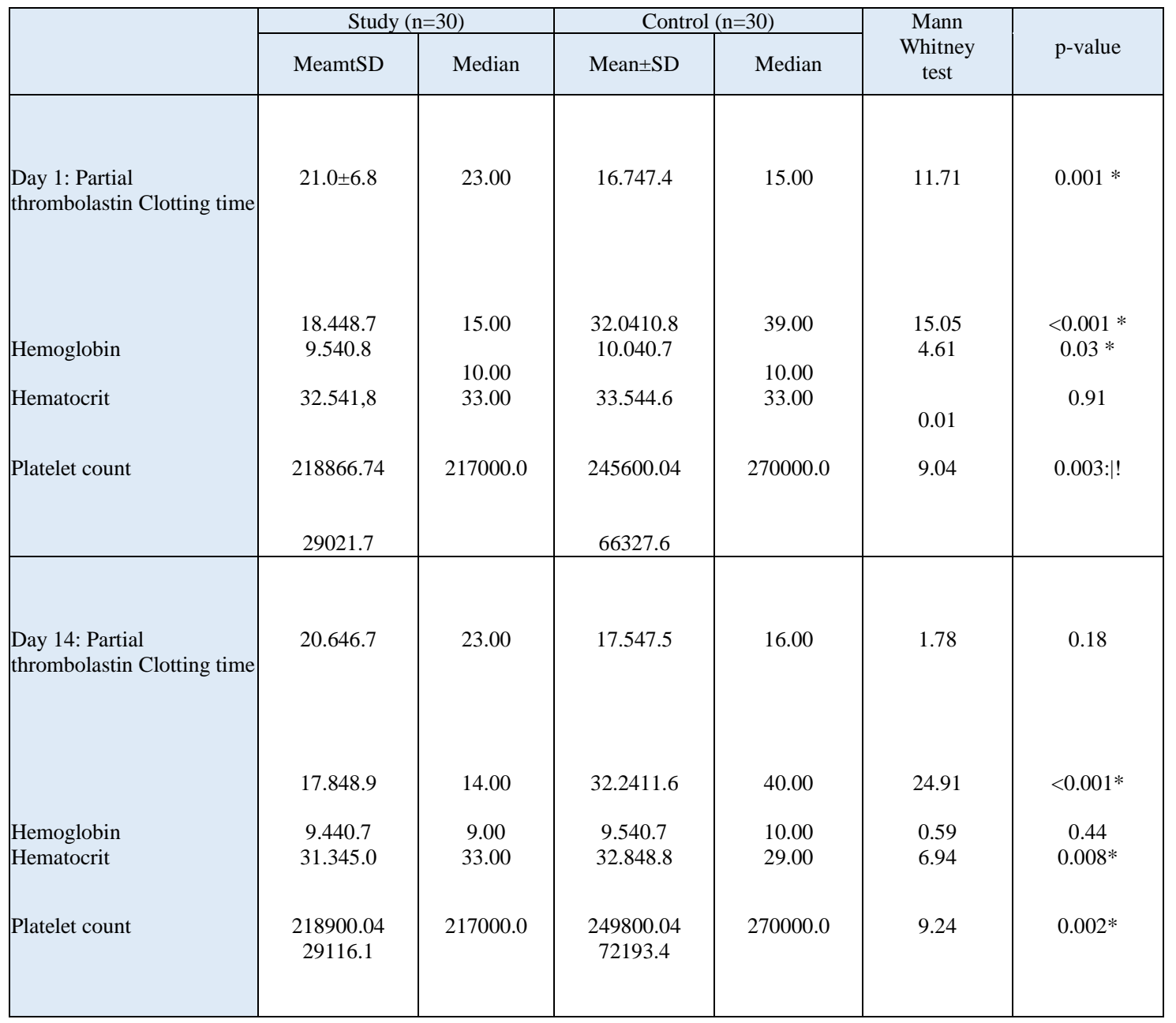

Table 6 demonstrates that all patients in the both groups had normal Doppler findings in both legs on days 1 and 14. Meanwhile, $2(6.7 \%)$ patients in the control group had DVT compared with none in the study group. However, the difference was not statistically significant $(\mathrm{p}=0.49)$.

Table 6: Comparison of Doppler results and DVT among patients in thestudy and control groups in the first and $14^{\text {lh }}$ days

\begin{tabular}{|c|c|c|c|c|c|c|}
\hline & \multicolumn{4}{|c|}{ Group } & \multirow{3}{*}{$\begin{array}{l}\mathrm{X} 2 \\
\text { test }\end{array}$} & \multirow{3}{*}{$\begin{array}{c}1> \\
\text { value }\end{array}$} \\
\hline & \multicolumn{2}{|c|}{$\begin{array}{c}\text { Study } \\
(\mathrm{n}=30)\end{array}$} & \multicolumn{2}{|c|}{$\begin{array}{l}\text { Control } \\
(\mathrm{n}=30)\end{array}$} & & \\
\hline & No. & $\%$ & No. & $\%$ & & \\
\hline \multicolumn{7}{|c|}{ Normal Doppler: Right leg: } \\
\hline Day 1 & 30 & 100.0 & 30 & 100.0 & 0.00 & 1.00 \\
\hline Day 14 & 30 & 100.0 & 30 & 100.0 & 0.00 & 1.00 \\
\hline \multicolumn{7}{|l|}{ Left leg: } \\
\hline Day 1 & 30 & 100.0 & 30 & 100.0 & 0.00 & 1.00 \\
\hline Dav 14 & 30 & 100.0 & 30 & 100.0 & 0.00 & 1.00 \\
\hline DVT: & & & & & & \\
\hline Absent & 30 & 100.0 & 28 & 93.3 & & \\
\hline Present & 0 & 0.0 & 2 & 6.7 & Fisher & 0.49 \\
\hline
\end{tabular}




\section{Discussion}

Deep Venous Thrombosis (DVT) is a potentially fatal complication (Pollack, 2011) \{1\}. It is responsible for most cases of venous thromboembolism Patients undergoing high-risk orthopedic procedures, specifically total hip arthroplasty, total knee arthroplasty, and hip fracture repair surgery are at a significantly increased risk without appropriate prophylaxis (Kamerkar et al., 2016) $\{7\}$

This study tried to evaluate the effect of implementing nursing care guidelines on the occurrence of deep vein thrombosis among orthopedic patients. The study hypothesized that occurrence of DVT would be reduced in orthopedic patients who are followed by evidence-based guidelines in comparison to orthopedic patients who are not followed by these guidelines. The study results indicate the success of the application of guidelines in reducing patients' risks of DVT, which leads to acceptance of the set research hypothesis

The patients assigned to the study and control groups of the present study were selected to have similar socio-demographic. This is of major importance since variables such as patient age, gender, as well as body weight may influence the risk of occurrence of DVT. In fact, the present study results revealed that the risk of development of DVT had a significant association with patient's age, with the risk increasing with older age. In line with this, a study in Turkey demonstrated that patients' age was a significant factor that increased the DVT risk among orthopedic surgery patients (Anderson et al, 2015) $\{\mathbf{8}\}$. On the same line, female gender was a significant predictor of DVT after orthopedic surgery among patients in Korea (Lee et al., 2015) $\{4\}$.

More importantly, the primary assessment of the patientsin the study and control groups of the current study revealedalmost no significant differences between them. Thus, themajority were at high risk of DVT, and were administered anticoagulants and salicylates. The effectiveness of these two types of medications has been documented in the pharmacological prophylaxis of DVT in major orthopedic surgery (Drescher et al., 2014) $\{9\}$.

In agreement with this foregoing present study finding, Liu et al. (2016)\{10\}in a study in China found that three out of 222 patients (2.25\%) developed DVT and/or thromboembolism. Another study in Japan (Fuji et al., 2016) $\{11\}$ comparing the effects of separate and combined mechanical and pharmacological preventive measures to mitigate the development of DVT in orthopedic patients reported that the incidence of DVT ranged between $6.0 \%$ and $13.0 \%$. Meanwhile, a study in China reported an incidence rate of $8.26 \%$ of DVT after orthopedic surgery

Therefore, the lower incidence of DVT among the patients in the study group of the current study is actually due to the combination of the two prophylactic measures, namely the pharmacologic approach through administration of anticoagulants and the mechanical approach through Compression Stockings and range of movement exercises. The anticoagulants act by suppressing the blood hypercoagulability while the CS act by increasing the venous blood flow velocity and impeding venous stasis in the legs. In congruence with this, a study in China found that active ankle y motion exercises may prevent the formation of lower-extremity DVT after orthopedic surgery through increasing maximum venous outflow and capacity, and by reducing blood rheology (Liu et al., 2016) $\{10\}$.

Nonetheless, the present study demonstrate dissimilarity significance between the patients in the study and control groups regarding their grade and score of DVT risk, with lower levels among those in the study group. The findings add to the evidence of the success of the guidelines in decreasing the risk of DVT among orthopedic high-risk patients. A similar success of combined pharmacological and mechanical prophylactic measures in decreasing the risk of DVT after orthopedic surgery was stated in a study in China (Jiang et al., 2015) $\{12\}$. In further support, and with a more objective evidence, the present study results demonstrated significantly better laboratory findings related to DVT risk among the patients in the study group. Thus, they had better values of thromboplastin time, clotting grade, clotting time, hemoglobin, platelet count, and hematocrit value. These laboratory results indicate a better blood clotting profile among the patients in the study group, with associated lower risk of DVT development. Such investigations are cornerstone in the close monitoring of patients at risk of DVT as they reflect the level of

Thromboembolic risk as well as the side effects of anticoagulants (Lee et al., 2015)\{4\}.An additional evidence of the effectiveness of the developed guidelines was the present study finding of the significant relation between nurses' performance of application of elastic stockings and range of motion exercise and patients' clotting grades. Thus, the better the nurse's performance, the lower is the patient's risk of DVT. A similar positive effect of a four-year quality improvement program to prevent inferior limb DVTs in intensive care unit in Italy was demonstrated, where the incidence of DVT dropped to $2.6 \%$ after training and involving nurses with direct application of DVT prophylaxis (Boddi et al., 2014)\{13\}. In congruence with this, Restrepo et al. (2015) \{14\}highlighted that an updated, clearly written guideline and an organization-wide procedure, with staff education, is sufficient to change practice and improve compliance. 


\section{Conclusion}

The application of the study intervention with the developed guidelines is effective in improving the erformance of all nurses in the study group in all steps of both procedures. The intervention is also beneficial in terms of patient outcomes, with significantly lower DVT risk grade and score compared with the control group patients

\section{Limitations}

Firstly, a major limitation of this study is the small sample size (due to their fear of surgery outcome) that limits the ability to generalize findings. However, results suggest clinical significance and the need to conduct future research with a larger sample size. Secondly, another limitation of this study is that patients are restricted in performing certain movements for a time period after surgery to avoid dislocating the joint replacement so this may influence patients' responses. Finally, a short period of follow-up considers another limitation for this study.

\section{Recommendation}

1. Patient's risk for developing deep vein thrombosis should be assessed by nurses constantly and progressively.

2. Simple booklet written in Arabic language should be developed and available for DVT prevention among orthopedic patients.

3. Educational program protocol or intervention should be conducted in orthopedic department for DVT prevention in order to enhance the quality of care and provide appropriate management of patients especially in the early postoperative period.

4. Long-term follow-up of patients who have undergone orthopedic surgeries patients is necessary to determine durability and long-term outcomes.

\section{Acknowledgements}

The authors are thankful to all patients who participated in this study also they appreciate the help provided by hospital staff in data collection and conduction of the study.

\section{References}

[1] Pollack, C. V. (2011). Advanced Management of Acute Iliofemoral Deep Venous Thrombosis: Emergency Department and Beyond. Annals of Emergency Medicine, 57: (6), 590-599

[2] Geerts, W. H., Bergqvist, D., Pineo, G. F., et al. (2008). Prevention of venous thromboembolism: American College of Chest Physicians evidence-based clinical practice guidelines. CHEST Journal, 133: (6_suppl), 381S-453S.Lunn

[3] warwick, D.( 2012). Prevention of venous thromboembolism in total knee and hip replacement. Circulation, 125: (17), 2151-2155

[4] Lee, S. Y., Ro, D. H., Chung, C. Y., et al. (2015.) Incidence of deep vein thrombosis after major lower limb orthopedic surgery: analysis of a nationwide claim registry. Yonsei medical journal, 56: (1), 139-145

[5] Won, Y. S., Kim, M., Jun, K. W., et al.( 2014). Incidence and Clinical Characteristics of Deep Vein Thrombosis (DVT) after Total Knee Arthroplasty (TKA) with DVT Chemoprophylaxis. World Journal of Cardiovascular Diseases, 4: (11), 531-538.

[6] Paffrath, T., Wafaisade, A., Lefering, R., et al.( 2010). Venous thromboembolism after severe trauma: Incidence, risk factors and outcome. Injury, 41: (1), 97-101

[7] Kamerkar, D. R., John, M. J., Desai, S. C., et al.( 2016). Arrive: A retrospective registry of Indian patients with venous thromboembolism. Indian journal of critical care medicine: peer-reviewed, official publication of Indian Society of Critical Care Medicine, 20: (3), 150

[8] Andersen, L., Husted, H., Otte, K. S., Gaarn-Larsen, L., and Kehlet, H.(,2011) Effect of high-dose preoperative methylprednisolone on pain and recovery after total knee arthroplasty: A randomized, placebo-controlled trial. British Journal of Anaesthesia, 106(2), 2011, 230-238.

[9] Drescher, F. S., Sirovich, B. E., Lee, A., et al.( 2014). Aspirin versus anticoagulation for prevention of venous thromboembolism major lower extremity orthopedic surgery: A systematic review and meta-analysis. Journal of hospital medicine, 9: (9), 579-585

[10] Liu, Z., Han, N., Xu, H., ( 2016). Incidence of venous thromboembolism and hemorrhage related safety studies of preoperative anticoagulation therapy in hip fracture patients undergoing surgical treatment: a case-control study. BMC Musculoskelet Disord, 17: (1), 76

[11] Fuji, T., Fujita, S., Kimura, T., et al. (2016). Clinical benefit of graduated compression stockings for prevention of venous thromboembolism after total knee arthroplasty: post hoc analysis of a phase 3 clinical study of edoxaban. Thromb J, 14: (1), 13.

[12] Jiang, Y., Li, J., Liu, Y., et al. 2015. Risk factors for deep vein thrombosis after orthopedic surgery and the diagnostic value of Ddimer. Annals of vascular surgery, 29: (4), 675-681.

[13] Boddi, M., Cecchi, A., Bonizzoli, M., et al. (2014). Follow-up after four-year quality improvement program to prevent inferior limb deep vein thrombosis in intensive care unit. Thrombosis Research, 134: (3), 578-583

[14] Restrepo, P., Jameson, D. \& Carroll, D. L. (2015). An evidence-based quality improvement project to improve deep vein thrombosis prophylaxis with mechanical modalities in the Surgical Intensive Care Unit. Journal of nursing care quality, 30: (1), 31. 